\title{
Principales miopatías: aproximación clínica, diagnóstico y manejo
}

\author{
Main myopathies: clinical approach, diagnosis and management
}

\author{
Virginia González Hidalgo \\ Servicio de Medicina Interna. Hospital Virgen del Puerto de Plasencia (Cáceres). España
}

\begin{abstract}
Myopathies make up an entity that, although rare, can have great clinical significance. These diseases can present a somewhat complex clinical and therapeutic approach due to the etiological versatility and the concomitant symptoms that can sometimes exist. The role of the clinical interview and physical examination is crucial in guiding our diagnostic suspicion. This article will present, in the first instance, the general approach to any type of myopathy in order to request the complementary tests required; and secondly, the clinical characteristics and entities that make up the main groups of myopathies, as well as their therapeutic approach.
\end{abstract}

Palabras clave: Miopatía; Creatin-quinasa; Biopsia muscular.

\section{INTRODUCCIÓN}

La sintomatología muscular es un hecho frecuente y común en muchas enfermedades sin que traduzcan daño muscular, sin embargo, pueden ser reflejo de una entidad en sí misma, denominándose en tal caso como miopatías. Las miopatías son un conjunto heterogéneo de desórdenes metabólicos, canalopatías o defectos estructurales que afectan al normal funcionamiento del músculo esquelético. A su vez, pueden clasificarse en hereditarias o adquiridas. Dentro de las primeras destacan las metabólicas, las mitocondriales y las canalopatías; las adquiridas incluyen entre otras, las endocrinas, las tóxicas o farmacológicas y las inflamatorias ${ }^{1,2}$.

\section{ORIENTACIÓN CLÍNICA Y DIAGNÓSTICA: HISTORIA CLÍNICA, EXPLORACIÓN FÍSICA Y PRUEBAS COMPLEMENTARIAS.}

Para iniciar el proceso diagnóstico es importante realizar una exhaustiva historia clínica y un minucioso examen físico, los cuales nos permitirán orientar y restringir nuestra sospecha clínica a un determinado grupo, y con ello, solicitar las pruebas complementarias de una forma más racional y dirigida para confirmar el diagnóstico.

El primer paso sería aproximarse al paciente que presenta debilidad muscular, intentando localizar el patrón que predomina; el siguiente buscar la etiología que subyace bajo dicho cuadro y así poder determinar la existencia, o no, de un tratamiento específico ${ }^{2,3}$.

\section{Historia clínica y exploración física \\ -Anamnesis}

La entrevista clínica es la primera toma de contacto que el médico tiene con el paciente afecto con una miopatía. Se considera una de las partes más importantes para orientar nuestra sospecha clínica, y dentro de las preguntas que formulemos, no deben omitirse las siguientes ${ }^{2,3}$ :

\section{RESUMEN}

Las miopatías conforman una entidad que, aunque poco frecuente, pueden tener gran trascendencia clínica. Estas pueden presentar un abordaje clínico y terapéutico algo complejo debido a la versatilidad etiológica y a la clínica concomitante que en ocasiones puede existir. El papel de la entrevista clínica y la exploración física es crucial de cara a orientar nuestra sospecha diagnóstica. Dentro del presente artículo se presentará, en primera instancia, el abordaje general de cualquier tipo de miopatía de cara a solicitar las pruebas complementarias requeridas; y en segundo lugar, las características clínicas y las entidades que conforman los principales grupos de miopatías, así como su abordaje terapéutico.

Keywords: Miopathy; Creatin-cinase; Muscular biopsy.

- ¿Qué es lo que significa para el paciente debilidad? A veces, ciertas condiciones como la anemia, la depresión, las infecciones o las enfermedades inflamatorias ocasionan en el paciente una condición de astenia o fatiga que nos la describen como debilidad.

- Temporalidad: Es importante determinar el inicio, la duración y la evolución del paciente con sintomatología muscular. La edad de inicio nos permite orientar si se pudiera tratar de una enfermedad hereditaria 0 adquirida. La evolución puede ser constante, lo que sugeriría con mayor probabilidad una forma adquirida, mientras que el carácter periódico, una hereditaria.

- Existencia de historia familiar de enfermedades musculares.

- Existencia de factor precipitante: interrogar si atribuyen la aparición de los síntomas a algún factor desencadenante (alimentos, deporte, algún fármaco o tóxico).

- La existencia de síntomas sistémicos.

\section{-Exploración física}

El patrón de debilidad más común tiende a involucrar simétricamente a la musculatura proximal de las extremidades superiores e inferiores ${ }^{4}$. Sin embargo, esto es un hecho inconstante, existiendo una serie de patrones descritos por Barohn el aR que nos permiten orientar la sospecha diagnóstica junto a los datos recabados de la anamnesis. El patrón más común, como se ha mencionado anteriormente, es el simétrico que involucra a la musculatura proximal de extremidades superiores e inferiores, también denominado distrofia de cinturas o distrofia muscular del anillo óseo. Dicho patrón se puede hallar tanto en miopatías hereditarias como adquiridas, siendo, por tanto, el más frecuente, pero el menos específico. Otro de los patrones descritos es la debilidad distal y simétrica, siendo ésta un hecho frecuente 
de las neuropatías. Otros menos frecuentes, pero para tener en cuenta, son la debilidad proximal de extremidades superiores y distal de miembros inferiores, presente en algunas miopatías hereditarias, y, el patrón de debilidad distal de miembros inferiores y proximal de miembros superiores, asimétrico, que como se verá más adelante, es patognomónico de la miositis por cuerpos de inclusión.

\section{Pruebas complementarias}

Una vez realizadas la anamnesis y la exploración física se procederá a la realización de una serie de pruebas complementarias, las cuales nos permitirán seguir orientando nuestra sospecha clínica y confirmarla mediante una biopsia muscular, la determinación de anticuerpos y/o la realización de test genéticos en el caso de las miopatías hereditarias.

\section{-Enzimas de daño muscular}

La medición de los niveles de enzimas de daño muscular es una parte importante de la evaluación de los pacientes con debilidad muscula o mialgias, y su monitorización es importante para valorar el curso de respuesta al tratamiento en algunas entidades.

-Creatinquinasa (CK): Es la enzima más empleada en el diagnóstico y el seguimiento de la enfermedad muscular, aunque no es un hallazgo específico de esta situación, pudiéndose ver elevados en situaciones como ejercicio físico, traumatismo 0 raza negra. Dicha enzima se encuentra localizada en la membrana mitocondrial, en las miofibrillas musculares y en el citoplasma muscular (de ahí que los niveles más elevados se observen en entidades como miopatías hereditarias, inflamatorias o por tóxicos, donde pueden presentarse valores superiores a 50 veces el valor normal). Sin embargo, en otras entidades como las neuropatías o las miopatías endocrinas, suelen presentar valores normales o muy levemente aumentados (excepto las producidas por hipotiroidismo), por lo que sus valores pueden ser también un modo de orientar la sospecha diagnóstica.

Si existen unos valores muy elevados de CK plasmático, se debe buscar la presencia de mioglobinuria, que puede conllevar a un daño renal.

-Aldolasa: La aldolasa se encuentra localizada en todos los tejidos, pero más frecuentemente a nivel muscular, hepático y cerebral. Sus niveles son menos específicos y sensibles que los de CK, aunque en algunos casos de sospecha de miopatías en que esta última presenta unos niveles normales, se puede encontrar una aldolasa elevada.

Otras enzimas de daño muscular que pueden hallarse elevadas son: lactato deshidrogenasa $(\mathrm{LDH}) 0$ las aminotransferasas (GOT y GPT) 1,4,5.

\section{-Electromiograma}

El electromiograma (EMG) debe ser considerado en todos los pacientes en que se sospeche la presencia de miopatía. La utilidad de los estudios neurofisiológicos radica en su alta sensibilidad y especificidad para el diagnóstico de miopatía y descartar otras causas de debilidad como pueden ser las ocurridas por alteraciones a nivel de la placa motora, entre otras. En las miopatías la conducción nerviosa suele ser normal, salvo en la miopatía por hipotiroidismo o por cuerpos de inclusión (como se verá más adelante). En esta prueba se deben explorar los músculos que presenten debilidad, y siempre de los miembros superiores e inferiores. Sin embargo, un EMG normal no nos excluye su existencia. Esta prueba, además, nos permite localizar qué musculo es más adecuado para biopsiar ${ }^{1,6}$.

\section{-Otras pruebas complementarias}

La biopsia muscular, los anticuerpos y/o test genéticos será los que nos confirmen el diagnóstico de sospecha en la mayoría de los casos, pero siempre junto a una anamnesis y una exploración física compatible, no de forma aislada. Esto es así debido a la existencia de patrones histológicos característicos y/0 anticuerpos específicos en algunas entidades.

\section{PRINCIPALES MIOPATÍAS}

\section{Miopatías inflamatorias}

Numerosos criterios han sido propuestos para la clasificación de este grupo de miopatías. Los más ampliamente utilizados han sido los que Bohan y Peter propusieron en 1975 para diagnosticar la poliomiositis (PM) y la dermatomiositis (DM), según los cuales, se incluían a pacientes que presentasen debilidad muscular proximal junto con una elevación de los niveles séricos de CK y un EMG compatible con miopatía, así como una biopsia muscular caracterizada por infiltrado inflamatorio ${ }^{7}$. Sin embargo, debido a los recientes avances moleculares con el descubrimiento de anticuerpos específicos y los hallazgos anatomopatológicos únicos en biopsias musculares, las sociedades reumatológicas europea y americana (EULAR/ACR) han auspiciado nuevos criterios en el año 2017, en los que se incluyen cinco entidades: DM, PM, miositis necrotizante autoinmune (MNA), miopatía por cuerpos de inclusión (MCI) y la miositis por solapamiento "overlap" (MS).

-Dermatomiositis: La DM es una miopatía que se caracteriza por la presencia de debilidad muscular simétrica y progresiva de predominio proximal junto a afectación cutánea. Dentro de la afectación dermatológica existen dos signos patognomónicos; las denominadas pápulas de Gottron (placas violáceas que se encuentran sobre las prominencias óseas de las articulaciones metacarpofalángicas e interfalánficas) y el eritema en heliotropo (eritema y edemas periorbitario). Aunque la coexistencia de ambas afectaciones es frecuente, existen situaciones en las que una u otra no están presentes. Cuando un paciente no aprecia síntomas musculares y las pruebas complementarias no reflejan daño del mismo, la enfermedad es conocida como dermatomiositis amiopática. En el caso de que el paciente no note sintomatología muscular, pero exista evidencia de daño muscular (elevación de CK, biopsia compatible...), en este caso la enfermedad pasaría a denominarse dermatomiositis hipomiopática. Sin embargo, también puede existir afectación muscular sin 
dermatológica, que sería la denominada dermatomiositis sin dermatitis.

Además de la clínica muscular y cutánea, estos pacientes presentan predisposición al desarrollo de enfermedad pulmonar intersticial, y un aumento de la incidencia tumoral de aproximadamente el $2 \%$ en los dos primeros años.

Los niveles de CK, se encuentran elevados, sin presentar clara correlación de los niveles con la gravedad de esta. Recientemente se ha descubierto la presencia de autoanticuerpos específicos, en los que presentan un patrón clínico único, que permite la realización de una subclasificación dentro de este grupo de miopatías.

A nivel histopatológico, la biopsia muscular se caracteriza por la presencia de atrofia perifascicular, hallazgo que, aunque no se encuentre en todos los casos, es muy específico. Además, presentan un infiltrado inflamatorio característico de estas entidades ${ }^{9,10,11}$.

-Miopatía necrotizante autoinmune: Se trata de un tipo de miopatía en la que los sujetos que la padecen presentan debilidad muscular simétrica de predominio proximal, siendo raras las manifestaciones extramusculares. Los niveles de CK en estos pacientes se encuentran muy elevados, siendo una de las condiciones que más elevación producen. En algunas ocasiones la elevación puede preceder a la clínica de debilidad. Aproximadamente dos tercios de los pacientes que la padecen presentan autoanticuerpos (antiSRP y anti-HMGCR). Existen diferencias entre los pacientes que presentan positividad para uno u otro autoanticuerpo. Así, los pacientes que presenten positividad para anti-SRP tendrán una debilidad muscular más marcada, presentarán mayor grado de necrosis en la biopsia muscular y una mayor tendencia a la afectación cardíaca. Sin embargo, en los pacientes que presentan anti-HMGCR, su presencia se puede deber a la toma de estatinas y asociarse a mayor predisposición para desarrollar una enfermedad pulmonar intersticial. En la biopsia muscular lo más característico es encontrar necrosis a nivel de las miofibrillas ${ }^{9,10,12}$.

-Miopatía esporádica por cuerpos de inclusión: Esta entidad presenta un patrón de debilidad de instauración progresiva, de predominio distal y asimétrico. A diferencia del resto de miopatías inflamatorias, presentan predisposición por el género femenino y por sujetos mayores de 50 años. En cuanto a los niveles plasmáticos de CK, presenta una leve elevación, no superando en la mayoría de las ocasiones valores superiores a los 1500 U/L. En cuanto al EMG, puede presentar un patrón mixto (afectación nerviosa y miopática), característica no compartida con el resto de las miopatías inflamatorias. El diagnóstico final vendrá dado por la biopsia muscular, en la que se puede observar histológicamente un patrón inflamatorio junto a la presencia de afectación mitocondrial, agregados inapropiados de proteínas y en algunos casos vacuolas ${ }^{9,13}$.

-Miositis de solapamiento "overlap": La miositis por solapamiento (MS) es un subtipo heterogéneo y poco reconocido de miopatías inflamatorias. Se considera que los pacientes que afectados por miositis más una característica de solapamiento clínico y/o de anticuerpo de otra enfermedad autoinmune, excluyendo la PM y DM presentan una MS. Dentro de ellas, la más representativa es el denominado síndrome antisintetasa (SA) que se caracteriza por la presencia de anticuerpos anti-ARS (anti-Jo1, anti-PL7, anti- PL12) junto a una 0 más de las siguientes características clínicas: miopatía inflamatoria, enfermedad intersticial pulmonar, artritis, síndrome de Raynaud, fiebre o la denominada "mano de mecánico" (presencia de lesiones hiperqueratósicas),

-Polimiositis: La PM se caracteriza por presentar debilidad muscular, aumento de las enzimas de daño muscular, un EMG compatible con miopatías e infiltrado inflamatorio en la biopsia muscular, sin otra clínica asociada de las descritas en las entidades anteriores. Por tanto se trataría de un diagnóstico de exclusión, y dichos pacientes deben ser estrechamente seguidos, pues puede tratarse de alguna de las miopatías anteriormente descritas que no hayan desarrollado otra clínica en el momento del diagnóstico9,10.

\section{Tratamiento de las miopatías inflamatorias}

Los objetivos del tratamiento son mejorar la fuerza muscular y evitar el desarrollo de complicaciones extramusculares.

Definir los regímenes de tratamiento óptimos para estos trastornos ha sido difícil debido a la rareza de estos, sus fenotipos altamente complejos y el número limitado de ensayos clínicos aleatorizados existentes. Los expertos recomiendan, en los casos de miopatías sin cuerpos de inclusión (DM, PM, MS, MNA) una terapia basada en corticoterapia como primera línea junto a tratamiento inmunosupresor.

El corticoide de elección en estos casos es la prednisona. En los casos leves y moderados se emplearán dosis de 0.5-1 $\mathrm{mg} / \mathrm{kg} / \mathrm{día}$ durante 4-6 semanas (con un máximo de dosis diaria de 80-100 mg/día). Mantener esta dosis más allá de las 6 semanas puede aumentar el riesgo de desarrollo de miopatía por corticoides. En aquellos casos graves esta pauta se verá precedida por bolos intravenosos de $0.5-1 \mathrm{~g} /$ día durante 3-5 días. Posteriormente, se mantendrá a dosis bajas durante 8-12 meses.

En cuanto a la inmunosupresión, salvo casos leves donde se puede omitir, los fármacos de primera línea son el metotrexate (MTX) o la azatioprina (AZA), prestando especial atención en los casos con afectación pulmonar intersticial y uso de MTX. Otros agentes útiles son el micofenolato o la ciclofosfamida.

En aquellos casos en que haya mala tolerancia o refractariedad al tratamiento de primera línea (corticoterapia e inmunosupresión) se podrá optar por tratamiento con inmunoglobulinas intravenosas (en perfusión a dosis de $2 \mathrm{mg} / \mathrm{kg} / \mathrm{mes}$ ) o Rituximab (anticuerpo monoclonal anti-CD20), 10,14

Sin embargo, miopatía por cuerpos de inclusión presenta refractariedad al tratamiento inmunosupresor y con glucocorticoides. En cuanto al uso de inmunoglobulinas, han mostrado 
efectividad en pacientes que presentan disfagia, mejorándola, pero con pobre respuesta a la debilidad muscular ${ }^{15}$. Recientes estudios han demostrado beneficios clínicos con Alentuzumab, anticuerpo monoclonal dirigido contra la proteína CD52. Dicho estudio tomó una biopsia muscular de 13 pacientes previas a la infusión de Alentuzumab a dosis de $1.2 \mathrm{mg} / \mathrm{kg}$ durante 4 días. Se re-biopsió a dichos pacientes a los seis meses, mostrando no solo una mejoría clínica, sino también un descenso del infiltrado inflamatorio ${ }^{16}$

\section{Miopatías tóxicas: miopatía por estatinas}

Los inhibidores de la HMGRC, más comúnmente conocidos como estatinas, son los fármacos más utilizados en el tratamiento del hipercolesterolemia, siendo empleado por aproximadamente el $25 \%$ de sujetos mayores de 65 años a nivel mundial ${ }^{17}$.

Hasta el año 2010, momento en que Christofer-Stine et al ${ }^{18}$ identificaron un tipo de miopatía asociada a la toma de estatinas (MAE), caracterizada por la presencia de necrosis en la biopsia (MNI), la única forma de MAE era la autolimitada no inmunomediada. Aunque el desarrollo de dicha miopatía suele acontecer a las pocas semanas del inicio del tratamiento hipolipemiante, no se puede descartar la posibilidad de su diagnóstico en sujetos en los que el tiempo de uso sea más prolongado. En cuanto a las características clínicas de cada uno de los dos grupos, las diferencias se basan fundamentalmente en la presencia de anticuerpos, biopsia característica y alteraciones electromiográficas más características de la forma con necrosis $\mathrm{s}^{19,20}$.

Para la evaluación de potenciales casos de una MAE, es decir, sujetos que presentan un cuadro de debilidad proximal y están en tratamiento hipolipemiante con estatinas se debe comenzar con la medición de los niveles de CK. En los casos en que estos niveles se encuentren por debajo de diez veces el límite superior de la normalidad ( $<10$ LSN) debe replantearse la posibilidad diagnóstica y buscar otras etiologías potenciales. Si por el contrario, los valores de esta enzima superan dicho límite (> 10 LSN), se procederá a la interrupción de la estatina y la reevaluación de dichos niveles en un periodo aproximado de 8 semanas (o antes si la sintomatología progresara). Si durante este periodo, los niveles han descendido (< 10 LSN) se procederá a evaluar el riesgo/ beneficio de su reintroducción; si por el contrario permanecen elevados, se solicitará el anticuerpo anti-HMG-CoA y en caso de positividad se hará el diagnóstico de presunción de MAE inmunomediada, estableciéndose un régimen terapéutico similar al de las miopatías inflamatorias ${ }^{21,22}$.

A diferencia de los pacientes que presentan una forma autolimitada de MEA, aquéllos que presentan una con anti HMG positivos no mejoran tras el cese del tratamiento. Éstos requerirán tratamiento farmacológico además de la retirada de las estatinas. Al igual que en el caso de las miopatías inflamatorias, no hay guía que recoja el tratamiento de en estos casos, aunque los expertos recomiendan un tratamiento similar a aquéllas. Tratamiento glucocorticoideo con prednisona a dosis de $1 \mathrm{~g} / \mathrm{kg} /$ día durante 4-6 semanas junto a un inmunosupresor, con posterior tratamiento de mantenimiento de corticoides ${ }^{21,22}$

\section{Miopatías endocrinas: miopatía por hipotiroidismo.}

El hipotiroidismo es una patología que puede cursar con síntomas y signos a diferentes niveles. La sintomatología muscular es una de las más frecuentes, produciendo entre otros síntomas mialgias, fatiga, calambres, debilidad muscular, etc. ${ }^{23}$ La miopatía por hipotiroidismo $(\mathrm{MH})$ es aquella condición en la que en presencia de hipotiroidismo los síntomas musculares predominan o es la única clínica presente en ausencia de sintomatología característica de hipotiroidis$\mathrm{mo}^{24}$.

Existen controversias en cuanto a la fisiopatología causante de la MH. Se contempla un papel importante de T3 y T4, pero no tanto de la TSH. El músculo obtiene T4 a partir de T3, haciendo posible un adecuado proceso de excitacióncontracción muscular. La T3 está involucrada en la expresión de cadenas de miosina (componente básico de las miofibrillas musculares), de las que hay dos isoformas: tipo 1, de conducción y contracción lenta, y tipo $2 \mathrm{~A}$ y $2 \mathrm{X}$, de conducción y contracción rápida. A nivel de las fibras tipo 1 , la condición hipotiroidea produce hipertrofia; mientras que a nivel de los tipos $2 \mathrm{~A}$ y $2 \mathrm{X}$, tiene lugar una atrofia y pérdida de estas. A nivel inmunohistoquímico, parece que hay una sobreexpresión de desmina, que hace que aparezca un perfil químico similar al que acontece en las enfermedades neuromusculares, conllevando a aparición de una alteración muscular neuromediada ${ }^{24,25}$.

Clínicamente esta entidad está caracterizada por una amplia sintomatología, entre las que se incluyen: mialgias, parestesias, síndrome del túnel carpiano o como en nuestro caso, debilidad y polineuropatía sensitivo-motora de predominio inferior ${ }^{4}$. A nivel analítico, puede producirse una elevación de CPK, la cual, presenta una correlación positiva con la gravedad del hipotiroidismo bioquímico (sus niveles se asocian positivamente con los de TSH, pero negativamente con los de T3 y T4), pero no así a nivel clínico. Estos niveles, en ocasiones, pueden precederse a la afectación muscular, por lo que, ante unos niveles elevados de dicha proteína en ausencia de etiología que pueda explicar dicho incremento, debe descartarse el hipotiroidismo como causa ${ }^{24,25}$

Estudios electromiográficos han demostrado una disminución en la duración de los potenciales motores. Sin embargo, esta prueba no debe emplearse como técnica diagnóstica, sino como apoyo al diagnóstico, dado que sólo en aproximadamente el $50 \%$ de los pacientes con MH se ve alterado ${ }^{23,24}$. El tratamiento consiste en la administración de levotiroxina con mejoría progresiva de la sintomatología y de la bioquímica, que puede ser parcial 0 completa ${ }^{25}$.

\section{Miopatías metabólicas}

Las miopatías metabólicas (MM) son enfermedades que afectan exclusiva o predominantemente al músculo esquelético y que son debidas a un déficit enzimático conocido, 
genéticamente determinado y causante de la enfermedad. La edad de presentación más frecuente en estas entidades son la pediátrica y juvenil, aunque pueden suceder a cualquier edad.

Las manifestaciones clínicas son variables, desde síntomas permanentes como la debilidad progresiva; síntomas episódicos con intolerancia al ejercicio, mialgias y/o mioglobinuria 0 una combinación de síntomas episódicos y permanentes.

Se clasifican en tres grandes grupos:

1. Miopatías por alteraciones del metabolismo del glucógeno (glucogenosis).

2. Miopatías por alteración del metabolismo lipídico. Estas enfermedades se caracterizan por la alteración del transporte de los ácidos grasos de cadena larga al interior de las mitocondrias, donde son metabolizados para proporcionar energía necesaria para el músculo. También puede afectar a otros tejidos, especialmente hígado, corazón y sistema nervioso central, dando cuadros clínicos diferentes.

3. Miopatías debidas a deficiencias de enzimas de la cadena respiratoria mitocondrial. Estas presentan una herencia materna y se presentan casi siempre en la edad adulta. A menudo la biopsia presenta de forma muy características las denominadas "fibras rojas-rasgadas".

El diagnóstico de este grupo heterogéneo de enfermedades se basa en la historia clínica con búsqueda de potenciales factores desencadenantes e historia familiar. Analíticamente aparecerá aumento de la CK, y finalizando habitualmente en estudio genético molecular.

Desde el punto de vista terapéutico, no existe tratamiento específico, por lo que se procederá a evitar los desencadenantes, asegurar adecuado aporte de glucosa y otros nutrientes, y en algunos casos muy seleccionados, el remplazo de la enzima deficiente ${ }^{1,26}$.

\section{Miopatía del paciente crítico}

La miopatía del paciente crítico (MPC) es una causa más frecuente de debilidad adquirida durante el ingreso en la Unidad de Cuidados Intensivos (UCI), pudiendo aparecer sola, o bien, en conjunto con la neuropatía de UCI. Los factores de riesgo a los que se asocian son fundamentalmente sepsis y fallo multiorgánico.

Clínicamente los pacientes presentan miembros fláccidos y débiles de forma simétrica y de predominio proximal, con reflejos musculares habitualmente normales y sin alteraciones sensitivas nerviosas. Los músculos que se afectan con mayor frecuencia son las extremidades y los músculos respiratorios, que van a producir un retraso en la retirada de la ventilación mecánica. Esto conlleva a un mayor tiempo de hospitalización y morbilidad.

A nivel del EMG presentan un patrón de disminución de la amplitud sin alteración en la latencia o velocidad de conducción. La biopsia muscular puede revelar pérdida de las cabezas de miosina o filamentos gruesos; además, puede haber necrosis, atrofia de las fibras II, hallazgos que pueden volver a la normalidad cuando la miopatía se ha recuperado 27,28,29.

\section{CONCLUSIONES}

Las miopatías incluyen un grupo heterogéneo de entidades etiológicas en cuya orientación diagnóstica juegan un papel importante la entrevista clínica y la exploración física. A veces, sus síntomas suelen considerarse triviales, pero es importante tener presente que bajo ellos pueden subyacer una entidad grave y de alta mortalidad, como es el caso de los tumores. Dada la diversidad etiológica y a la posibilidad de afectación multiorgánica es importante un abordaje multidisciplinar por parte de médicos generalistas, como los médicos internistas.

\section{CONFLICTS OF INTEREST AND SOURCE OF FUNDING}

The author declares that there is no conflict of interest in this work. Financiación: The author declares that there were no external sources of study for the performance of this article. Aspectos éticos: The author declares that no data that allows identification of the patient appears in this article.

\section{BIBLIOGRAFÍA}

1. Domingo-Horne RM, Salajegheh MK. An approach to myophaty for the primary care clinician.2018; 131 (3), 237-43.

2. Barohn RJ, Dimachkie MM, Jackson CE. A pattern recognition approach to patients with a suspected myopathy. Neurol Clin. 2014; 32(3):1-42.

3. Milisenda JC, Grau Junyent JM. Protocolo diagnóstico de las miopatías. Medicine. 2017; 12 (29): 1725-8

4. Suresh E, Wimalaratna S. Proximal myopathy: Diagnostic approach and initial management. Postgrad Med J. 2013; 89 (1054), 470-77.

5. Miller ML. Muscle enzymes in the evaluation of neuromuscular diseases. Targoff IN, Shefner JM, Ramirez Curtis M (ed). Whaltman, MA, Uptodate Inc. https://www. uptodate.com/contents/muscle-enzymes-in-the-evaluation-of-neuromusculardiseases.

6. Gutiérrez-Gutiérrez G, Barbosa López C, Navacerrada F, Miralles Martínez A. Utilidad del electromiograma en el diagnóstico de miopatías inflamatorias. Reumatol Clin. 2012; 8 (4): 195-200

7. Bohan A, Peter JB. Polymiositis and dermatomiositis. N Eng J Med, 1975.292: 344-47.

8. Lumberg IE, Tjarnlund A, Bottaii M, Werth VP, Pilkington C, de Visser M et al. European League Against Rheumatism/American Collegue of Rheumatology classification criteria for adults and juvenile idiopathic inflammatory myopathies and their major subgroups. Ann Rheum Dis. 2017; 76: 1995-64.

9. Trallero.Araguás E, Milisenda JC, Grau-Junyent JM, Mammen AL. Classification and management of adult inflammatory myopathies. Lancet Neurol. 2018; 17 (9): 816-28.

10. Mammen AL. Autoimmune Myopathies. Lifelong Learning in Neurology. 2016; 22(6), 1852-70

11. Findlay AR, Goyal NA, Mozzafar T. An overview of poymyositis and dermatomiositis. Muscle Nerve. 2015; 51: 638-56.

12. Pinal-Fernandez I, Casal-Dominguez M, Mammen AL. Immune-Mediated Necrotizing Myopathy. Curr Rheum Rep. 2018; 20(4); 1-19.

13. Weihl CC. Sporadic Inclusion Body Myositis and Other Rimmed Vacuolar myopathies. Neurm Junct Dis. 2019: 1586-98.

14. Mandel DE, Malemud CJ, Askari AD. Idiopathic inflammatory myopathies: a review of the classification and impact of pathogenesis. Int J Mol Sci. 2017; 18 (5):1-10.

15. Oddis C. Update on the pharmacological treatment of adults miositis. J.Inter Med. 2016;280; 63-74.

16. Schmit K, Kleinschinitz K, Rakocevi G, Dalakas MC, Schmidt J. Molecular treatment effects of alemtuzumab in skeletal muscles of patients with IBM. BMC Neurology. 2016;16:48.

17. Wallach-Hildemoes H, Stovring H, Holme Hansen E, Howse K, Pétursson H. Statin prescribing acording to gender, age and indication: what about the benefit-risk balance?- J Eval Clin Pract. 2016; 22: 235-46.

18. Christopher-Stine L, Casciola-Rosen LA, Hong G et al. A novel autoantibody recognizing 200-kd and 100-kd protein is associated with an immune-mediated necrotizing myopathy. Arthritis Rheum. 2011; 62:2757-66.

19. Babu S, Li Y. Statin induced necrotitizing autoinmune myopathy. J Neurl Sci. 2015; 351:13-7.

20. Christopher-Stine L, Basharat P. Statin-associated immune-mediated myopathy: 
biology and clinical implications. Current Opinion in Lipidology. 2017; 28 (2), 186-

92.

21. Mammen AL. Statin-Associated Autoimmune Myopathy. New Eng J Med.

2016;374:664-9.

22. Pasnoor M, Barohn RJ, Dimachkie MM. Toxic myopathies. implications.Current Opinion in Lipidology. 2018; 31: 575-82.

23. Eslamian F, Bahrami A, Aghamohammadzadeh N, Niafar M, Selekzamini Y, Behkamrad K. Electrophysiologic changes in patients with untreated primary hypothyroidism. J clin Neurophysiol. 2011; 28 (3): 323-8.

24. Sindoni A, Rodolico C, Pappalardo MA, Portaro S, Benvega S. Hypothyroid myopathy: A peculiar clinical presentation of thyroid failure. Review of the literatura. Rev End Metab Disord. 2016; 17:499.

25. Miller ML, Rubin DI. Hypothyroid myopathy. Aminoff, MJ, Shefner JM, Ross DS Wilterdink, JL (ed). Waltham, MA: UpToDate Inc. https://www.uptodate.com/ contents/hypothyroid-myopathy

26. Darras BT. Approach to the metabolic myopathies. Patterson MC, Dashe JF (ed), Whaltman, MA; Uptodate Inc. https://www.uptodate.com/contents/approach-tothe-metabolic-myopathies.

27. Lacomis D. Neuromuscular weakness related to critical illness. Shefner JM, Eichler AF (ed), Whaltman, MA; Uptodate Inc. https://www.uptodate.com/contents/ neuromuscular-weakness-related-to-critical-illness.

28. Stevens RD, Marshall SA, Cornblath DR et al. A framework for diagnosis and classifying intensive care unit-acquired weakness. Cut Care Med. 2009; 37: S299308.

29. Farmer JC, Griffiths RD. UCl-aquired weakness: Proceedings of a Round Table Conference in Brussels, Belgium. Crit Care Med. 2009; 37 